Supporting Information for:

\title{
Toward Effect of Accelerators on the Curing of Unsaturated Polyester Resins: Kinetic Model for Room Temperature Curing.
}

Eliška Matušková,† Jaromír Vinklárek,t and Jan Honzíček *,†

†Institute of Chemistry and Technology of Macromolecular Materials, Faculty of Chemical Technology, University of Pardubice, Studentská 573, 53210 Pardubice, Czech Republic \$Department of General and Inorganic Chemistry, Faculty of Chemical Technology, University of Pardubice, Studentská 573, 53210 Pardubice, Czech Republic

P. 2 Table S1. Gelation times of the UP/STY/MEKP system.

P. 2 Table S2. Exothermic behavior of the UP/STY/MEKP system.

P. 3 Table S3. Coloration parameters of the UP/STY/MEKP system treated with given accelerator.

P. 3 Figure S1. Exothermic behavior of the UP/STY/MEKP/VO-A system.

P. 4 Figure S2. Conversion profiles for UP/STY/MEKP/Fe-O and UP/STY/MEKP/Fe-H.

P. 4 Figure S3. Conversion profiles for UP/STY/MEKP/VO-T.

P. 5 Figure S4. Rates of vinylene and styrene consumption for UP/STY/MEKP/Co-C.

P. 5 Figure S5. Rates of vinylene and styrene consumption for UP/STY/MEKP/Fe-H and $\mathrm{UP} / \mathrm{STY} / \mathrm{MEKP} / \mathrm{Fe}-\mathrm{O}$.

P. 6 Figure S6. Rates of vinylene and styrene consumption for UP/STY/MEKP/VO-T. 
Table S1. Gelation times of the UP/STY/MEKP system treated with given accelerator.

\begin{tabular}{|c|c|c|c|c|c|c|c|c|}
\hline & $\begin{array}{l}\text { Conc. } \\
\text { (wt.\%) }\end{array}$ & $\begin{array}{c}t_{\mathrm{gel}} \\
(\mathrm{min})\end{array}$ & & $\begin{array}{l}\text { Conc. } \\
\text { (wt.\%) }\end{array}$ & $\begin{array}{c}t_{\mathrm{gel}} \\
(\mathrm{min})\end{array}$ & & $\begin{array}{l}\text { Conc. } \\
\text { (wt.\%) }\end{array}$ & $\begin{array}{c}t_{\mathrm{gel}} \\
(\mathrm{min})\end{array}$ \\
\hline \multirow{7}{*}{ نُ } & 0.1 & $3.5 \pm 0.2$ & & $1 \times 10^{-3}$ & $6.4 \pm 0.3$ & \multirow{7}{*}{ 吾 } & 0.01 & $2.68 \pm 0.05$ \\
\hline & 0.06 & $3.9 \pm 0.1$ & & $6 \times 10^{-4}$ & $5.4 \pm 0.1$ & & 0.006 & $3.3 \pm 0.2$ \\
\hline & 0.03 & $5.3 \pm 0.1$ & & $3 \times 10^{-4}$ & $5.7 \pm 0.2$ & & 0.003 & $5.4 \pm 0.2$ \\
\hline & 0.01 & $9.8 \pm 0.2$ & j & $1 \times 10^{-4}$ & $8.0 \pm 0.4$ & & $1 \times 10^{-3}$ & $11.2 \pm 0.5$ \\
\hline & 0.006 & $16.5 \pm 0.9$ & & $6 \times 10^{-5}$ & $10.4 \pm 0.5$ & & $6 \times 10^{-4}$ & $17.3 \pm 1.2$ \\
\hline & 0.003 & $31.5 \pm 2.0$ & & $3 \times 10^{-5}$ & $15.6 \pm 0.3$ & & $3 \times 10^{-4}$ & $32.3 \pm 3.0$ \\
\hline & $1 \times 10^{-3}$ & $108.0 \pm 8.4$ & & $1 \times 10^{-5}$ & $48.6 \pm 3.2$ & & $1 \times 10^{-4}$ & $156.4 \pm 10.8$ \\
\hline \multirow{7}{*}{$\begin{array}{l}0 \\
0\end{array}$} & 0.01 & $63.0 \pm 1.5$ & & 0.03 & $3.8 \pm 0.1$ & \multirow{7}{*}{$\stackrel{H}{\circ}$} & 0.01 & $3.2 \pm 0.2$ \\
\hline & 0.006 & $25.9 \pm 0.5$ & & 0.01 & $6.2 \pm 0.2$ & & 0.006 & $5.3 \pm 0.3$ \\
\hline & 0.003 & $32.3 \pm 0.7$ & $\varangle$ & 0.006 & $7.2 \pm 0.1$ & & 0.003 & $8.0 \pm 0.4$ \\
\hline & $1 \times 10^{-3}$ & $30.6 \pm 0.4$ & $0^{\prime}$ & 0.003 & $10.4 \pm 0.6$ & & $1 \times 10^{-3}$ & $13.6 \pm 1.5$ \\
\hline & $6 \times 10^{-4}$ & $37.9 \pm 1.9$ & $>$ & $1 \times 10^{-3}$ & $21.3 \pm 1.1$ & & $6 \times 10^{-4}$ & $16.7 \pm 1.1$ \\
\hline & $3 \times 10^{-4}$ & $71.2 \pm 6.3$ & & $3 \times 10^{-4}$ & $50.3 \pm 2.2$ & & $3 \times 10^{-4}$ & $41.7 \pm 6.2$ \\
\hline & $1 \times 10^{-4}$ & $155.1 \pm 11.5$ & & $1 \times 10^{-4}$ & $156.9 \pm 16.2$ & & $1 \times 10^{-4}$ & $131.9 \pm 18.6$ \\
\hline
\end{tabular}

Table S2. Exothermic behavior of the UP/STY/MEKP system treated with given accelerator.

\begin{tabular}{|c|c|c|c|c|c|c|c|c|c|}
\hline & $\begin{array}{l}\text { Conc. } \\
\text { (wt.\%) }\end{array}$ & $\begin{array}{l}\Delta T_{\mathrm{ad}} \\
\left({ }^{\circ} \mathrm{C}\right)\end{array}$ & $\begin{array}{c}\left(\mathrm{d} T_{\mathrm{ad} /} \mathrm{d} t\right)_{\max } \\
\left({ }^{\circ} \mathrm{C}\right)^{a}\end{array}$ & $\begin{array}{c}t_{\max } \\
(\min )^{a}\end{array}$ & & $\begin{array}{l}\text { Conc. } \\
\text { (wt.\%) }\end{array}$ & $\begin{array}{l}\Delta T_{\mathrm{ad}} \\
\left({ }^{\circ} \mathrm{C}\right)\end{array}$ & $\begin{array}{c}\left(\mathrm{d} T_{\mathrm{ad}} / \mathrm{d} t\right)_{\max } \\
\left({ }^{\circ} \mathrm{C}\right)\end{array}$ & $\begin{array}{c}t_{\max } \\
(\min )\end{array}$ \\
\hline \multirow{7}{*}{$\begin{array}{l}0 \\
\dot{1} \\
0\end{array}$} & 0.1 & 98.7 & $18.1(8.3)$ & $8.1(10.3)$ & \multirow{7}{*}{$\begin{array}{c}0 \\
1 \\
1 \\
1\end{array}$} & $1 \times 10^{-3}$ & 144.9 & $(4.0) 65.0$ & (5.4) 12.3 \\
\hline & 0.06 & 115.7 & $27.6(23.9)$ & $8.6(9.8)$ & & $6 \times 10^{-4}$ & 157.9 & (23.6) 71.0 & (8.0) 10.0 \\
\hline & 0.03 & 126.2 & 44.7 & 9.3 & & $3 \times 10^{-4}$ & 154.2 & 64.9 & (7.5) 9.6 \\
\hline & 0.01 & 134.4 & 70.9 & 10.3 & & $1 \times 10^{-4}$ & 152.0 & (20.4) 56.0 & 13.0 \\
\hline & 0.006 & 138.2 & 88.0 & 11.6 & & $6 \times 10^{-5}$ & 149.0 & 38.1 & 16.7 (12.8) \\
\hline & 0.003 & 143.8 & 75.8 & 23.3 & & $3 \times 10^{-5}$ & 141.9 & (7.1) 13.4 & 27.0 \\
\hline & $1 \times 10^{-3}$ & 153.3 & 20.8 & 101.7 & & $1 \times 10^{-5}$ & n.d. ${ }^{b}$ & 1.2 & 69.0 \\
\hline \multirow{7}{*}{$\begin{array}{l}\frac{T}{1} \\
\text { d. }\end{array}$} & 0.01 & 80.4 & $64.5(10.4)$ & $3.1(4.6)$ & \multirow{7}{*}{$\begin{array}{l}1 \\
0 \\
1\end{array}$} & 0.01 & 145.6 & 69.9 & 36.2 \\
\hline & 0.006 & 102.0 & $86.5(24.6)$ & $4.2(5.1)$ & & 0.006 & 147.9 & 82.9 & 17.1 \\
\hline & 0.003 & 108.9 & 95.0 & 5.0 & & 0.003 & 148.2 & 97.9 & 30.4 \\
\hline & $1 \times 10^{-3}$ & 144.2 & 159.0 & 9.1 & & $1 \times 10^{-3}$ & 147.2 & 54.2 & 32.7 \\
\hline & $6 \times 10^{-4}$ & 148.4 & 131.0 & 13.9 & & $6 \times 10^{-4}$ & 153.2 & 45.2 & 52.2 \\
\hline & $3 \times 10^{-4}$ & 153.8 & 91.0 & 24.4 & & $3 \times 10^{-4}$ & 149.4 & 24.3 & 78.1 \\
\hline & $1 \times 10^{-4}$ & 150.8 & 25.3 & 58.9 & & $1 \times 10^{-4}$ & 146.9 & 6.2 & 143.6 \\
\hline \multirow{7}{*}{$\stackrel{⿱}{\circ}$} & 0.06 & 150.2 & $(\text { n.d. })^{b} 14.3$ & $(<1) 68.5$ & \multirow{7}{*}{ 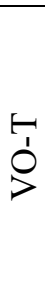 } & 0.01 & 138.6 & 89.6 & 5.4 \\
\hline & 0.03 & 139.6 & 24.9 & (3.0) 16.6 & & 0.006 & 136.7 & 83.4 & 6.6 \\
\hline & 0.01 & 131.8 & (92.9) 51.8 & (8.1) 8.8 & & 0.003 & 146.4 & 83.9 & 9.38 \\
\hline & 0.006 & 142.0 & 89.6 & 12.1 & & $1 \times 10^{-3}$ & 147.4 & 69.1 & 17.4 \\
\hline & 0.003 & 145.3 & 85.6 & 12.5 & & $6 \times 10^{-4}$ & 151.0 & 63.5 & 20.7 \\
\hline & $1 \times 10^{-3}$ & 150.8 & 46.7 & 49.1 & & $3 \times 10^{-4}$ & 152.8 & 56.0 & 28.3 \\
\hline & $6 \times 10^{-4}$ & 151.2 & 35.3 & 58.9 & & $1 \times 10^{-4}$ & 138.0 & 7.2 & 152.8 \\
\hline
\end{tabular}

${ }^{a}$ secondary peaks at derivative curves are given in parentheses; ${ }^{b}$ not determined. 
Table S3. Coloration parameters of the UP/STY/MEKP system treated with given accelerator.

\begin{tabular}{|c|c|c|c|c|c|c|c|c|c|}
\hline & $\begin{array}{l}\text { Conc. } \\
\text { (wt.\%) }\end{array}$ & $\mathrm{L}$ & $a^{*}$ & $b^{*}$ & & $\begin{array}{l}\text { Conc. } \\
\text { (wt.\%) }\end{array}$ & $\mathrm{L}$ & $a^{*}$ & $b^{*}$ \\
\hline \multirow{7}{*}{ ن̀ } & 0.1 & $75.8 \pm 1.5$ & $4.51 \pm 0.34$ & $7.33 \pm 0.85$ & \multirow{7}{*}{$\begin{array}{l}0 \\
1 \\
1 \\
\text { I. }\end{array}$} & $1 \times 10^{-3}$ & $89.6 \pm 1.9$ & $-2.24 \pm 0.08$ & $6.23 \pm 0.57$ \\
\hline & 0.06 & $81.5 \pm 2.4$ & $2.34 \pm 0.15$ & $5.88 \pm 0.28$ & & $6 \times 10^{-4}$ & $89.2 \pm 2.6$ & $-1.44 \pm 0.14$ & $4.94 \pm 0.76$ \\
\hline & 0.03 & $83.9 \pm 2.1$ & $1.17 \pm 0.24$ & $3.60 \pm 0.29$ & & $3 \times 10^{-4}$ & $90.5 \pm 1.7$ & $-0.76 \pm 0.07$ & $2.64 \pm 0.50$ \\
\hline & 0.01 & $89.2 \pm 2.4$ & $0.40 \pm 0.21$ & $2.16 \pm 0.38$ & & $1 \times 10^{-4}$ & $92.6 \pm 2.4$ & $-0.43 \pm 0.04$ & $1.91 \pm 0.25$ \\
\hline & 0.006 & $91.7 \pm 1.3$ & $0.21 \pm 0.10$ & $1.64 \pm 0.14$ & & $6 \times 10^{-5}$ & $92.4 \pm 2.1$ & $-0.41 \pm 0.09$ & $1.97 \pm 0.30$ \\
\hline & 0.003 & $91.7 \pm 1.7$ & $0.04 \pm 0.06$ & $1.35 \pm 0.35$ & & $3 \times 10^{-5}$ & $92.6 \pm 1.8$ & $-0.23 \pm 0.08$ & $2.08 \pm 0.45$ \\
\hline & $1 \times 10^{-3}$ & $91.6 \pm 0.3$ & $-0.05 \pm 0.09$ & $1.31 \pm 0.18$ & & $1 \times 10^{-5}$ & $91.0 \pm 1.5$ & $-0.16 \pm 0.06$ & $1.98 \pm 0.43$ \\
\hline \multirow{8}{*}{$\begin{array}{l}\text { T. } \\
\text { d } \\
\text { I. }\end{array}$} & 0.01 & $25.8 \pm 1.0$ & $20.0 \pm 0.4$ & $36.8 \pm 1.1$ & \multirow{8}{*}{$\stackrel{0}{0}$} & 0.003 & $92.8 \pm 3.1$ & $-2.39 \pm 0.14$ & $6.66 \pm 0.36$ \\
\hline & 0.006 & $42.1 \pm 1.6$ & $16.8 \pm 0.4$ & $41.7 \pm 1.1$ & & $1 \times 10^{-3}$ & 94.4 & $-0.58 \pm 0.10$ & $3.41 \pm 0.17$ \\
\hline & 0.003 & $66.3 \pm 0.7$ & $6.12 \pm 0.29$ & $32.2 \pm 0.3$ & & $6 \times 10^{-4}$ & 92.9 & -0.3 & $2.10 \pm 0.40$ \\
\hline & $1 \times 10^{-3}$ & $79.3 \pm 1.1$ & $1.47 \pm 0.13$ & $22.2 \pm 0.3$ & & $3 \times 10^{-4}$ & 93.7 & -0.32 & $1.50 \pm 0.25$ \\
\hline & $6 \times 10^{-4}$ & $89.3 \pm 1.4$ & $-0.79 \pm 0.07$ & $10.6 \pm 0.2$ & & $1 \times 10^{-4}$ & $93.7 \pm 1.9$ & $-0.33 \pm 0.07$ & $1.36 \pm 0.25$ \\
\hline & $3 \times 10^{-4}$ & $91.6 \pm 1.0$ & $-0.74 \pm 0.09$ & $6.14 \pm 0.17$ & & & & & \\
\hline & $10^{-4}$ & $93.4 \pm 0.9$ & $-0.56 \pm 0.06$ & $4.01 \pm 0.16$ & & & & & \\
\hline & $6 \times 10$ & $93.6 \pm$ & -0.4 & 2.62 & & & & & \\
\hline \multirow{7}{*}{$\stackrel{1}{0}$} & & $90.4 \pm 3.1$ & $-3.36 \pm 0.34$ & 12.9 & \multirow{7}{*}{ 보 } & 0.0 & 89.5 & -2.6 & $8.13 \pm 0.66$ \\
\hline & 006 & $90.1 \pm 5.7$ & $-2.70 \pm 0.39$ & $11.3 \pm 0.9$ & & 0.006 & $90.7 \pm 3.5$ & $-2.20 \pm$ & $6.79 \pm 0.68$ \\
\hline & 0.003 & $92.3 \pm 2.8$ & $-1.14 \pm 0.19$ & $6.68 \pm 0.56$ & & 0.003 & $92.5 \pm 2.7$ & $-1.74 \pm 0.08$ & $5.51 \pm 0.21$ \\
\hline & $1 \times 10^{-3}$ & $92.9 \pm 1.4$ & $-0.47 \pm 0.09$ & $3.70 \pm 0.22$ & & $1 \times 10^{-3}$ & $94.3 \pm 1.2$ & $-0.32 \pm 0.05$ & $3.02 \pm 0.15$ \\
\hline & $6 \times 10^{-4}$ & $94.2 \pm 1.5$ & $-0.37 \pm 0.07$ & $2.81 \pm 0.14$ & & $6 \times 10^{-4}$ & $93.7 \pm 1.4$ & $-0.31 \pm 0.06$ & $2.59 \pm 0.22$ \\
\hline & $3 \times 10^{-4}$ & $93.9 \pm 0.8$ & $-0.34 \pm 0.11$ & $2.56 \pm 0.11$ & & $3 \times 10^{-4}$ & $93.8 \pm 1.3$ & $-0.33 \pm 0.14$ & $1.87 \pm 0.25$ \\
\hline & $1 \times 10^{-4}$ & $95.0 \pm 1.4$ & $-0.34 \pm 0.12$ & $1.90 \pm 0.20$ & & $1 \times 10^{-4}$ & $94.5 \pm 1.5$ & $-0.25 \pm 0.04$ & $1.58 \pm 0.17$ \\
\hline
\end{tabular}

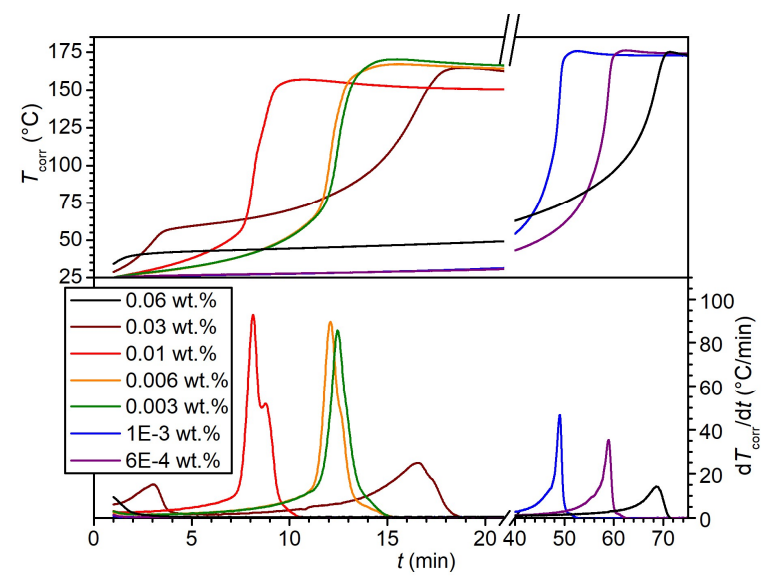

Figure S1. Effect of metal concentration on corrected exotherms (top) and rate of temperature rise (bottom) for UP/STY/MEKP/VO-A. 

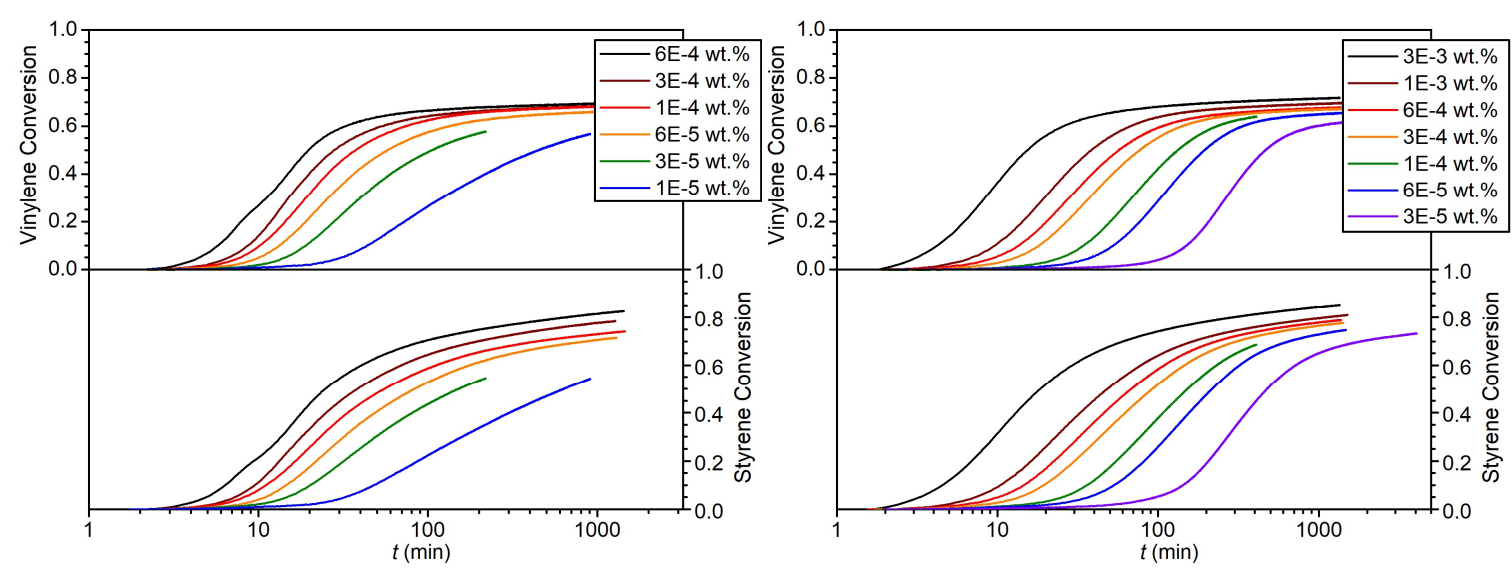

Figure S2. Vinylene and styrene conversion profiles for UP/STY/MEKP/Fe-O (left) and $\mathrm{UP} / \mathrm{STY} / \mathrm{MEKP} / \mathrm{Fe}-\mathrm{H}$ (right) at isothermal conditions.

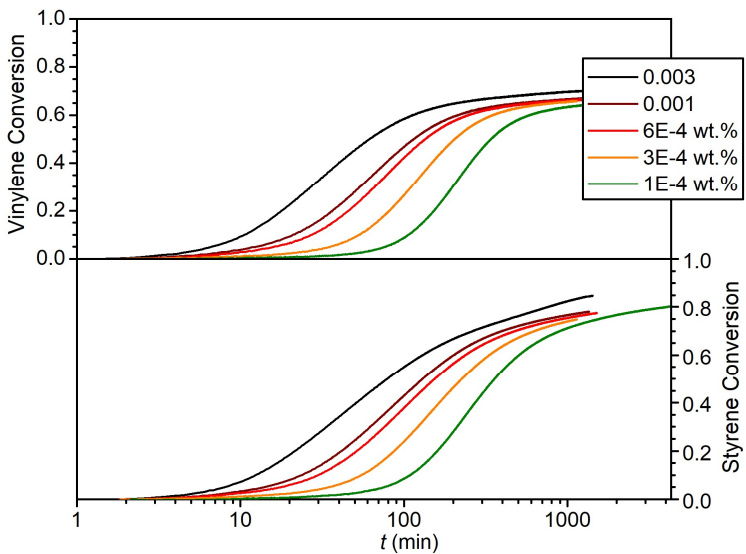

Figure S3. Vinylene and styrene conversion profiles for UP/STY/MEKP/VO-T at isothermal conditions. 

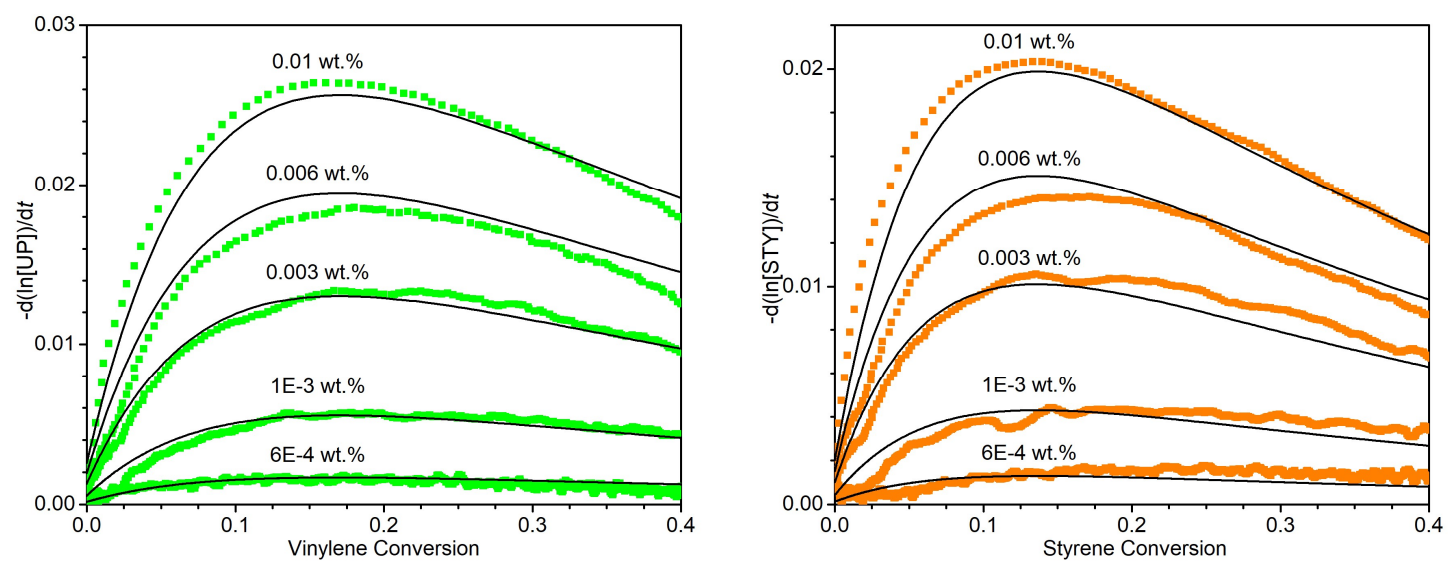

Figure S4. Rates of polyester vinylene (left) and styrene (right) consumption at different dosage of the Co-C. Experimental data (color squares) and according to Eqs. 9 (black lines).
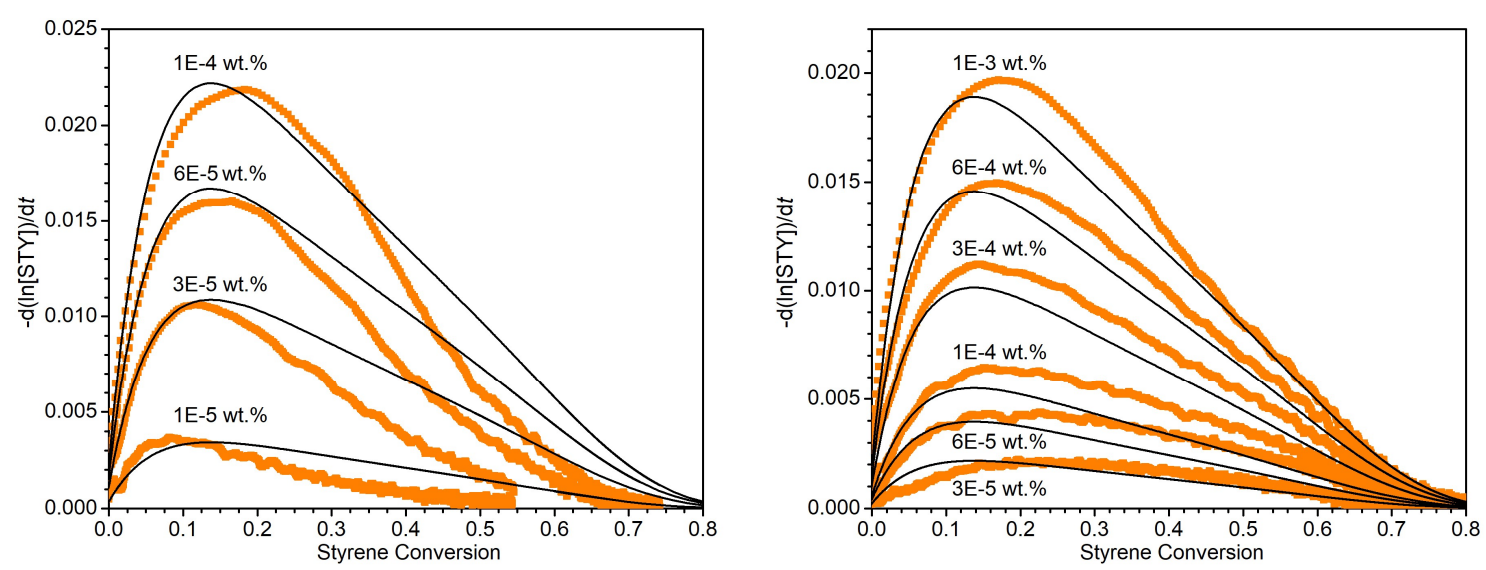

Figure S5. Rates of styrene consumption at different dosage of Fe-O (left) and Fe-H (right).

Experimental data (orange squares) and prediction according to Eq. 11b (black lines). 


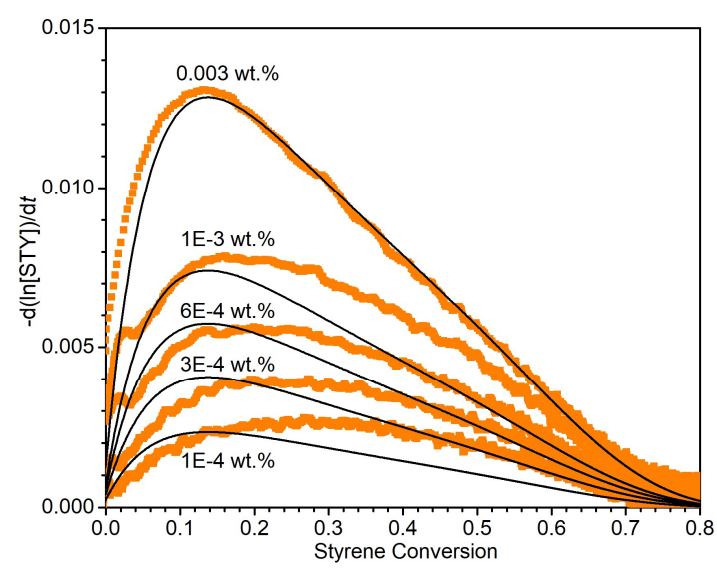

Figure S6. Rates of styrene consumption at different dosage of VO-T. Experimental data (orange squares) and prediction according to Eq. 11b (black lines). 